SUBJECT AREAS:

MICROBIOLOGY

TECHNIQUES

BIOTECHNOLOGY

Received

30 July 2014

Accepted

22 October 2014

Published

7 November 2014

Correspondence and requests for materials should be addressed to Y.U. (yum@kist.re.kr)

\section{Electricity-driven metabolic shift through direct electron uptake by electroactive heterotroph Clostridium pasteurianum}

\author{
Okkyoung Choi, Taeyeon Kim, Han Min Woo \& Youngsoon Um
}

Clean Energy Research Center, National Agenda Research Division, Korea Institute of Science and Technology (KIST), Seongbuk-gu, Seoul 136-791, South Korea.

Although microbes directly accepting electrons from a cathode have been applied for $\mathrm{CO}_{2}$ reduction to produce multicarbon-compounds, a high electron demand and low product concentration are critical limitations. Alternatively, the utilization of electrons as a co-reducing power during fermentation has been attempted, but there must be exogenous mediators due to the lack of an electroactive heterotroph. Here, we show that Clostridium pasteurianum DSM 525 simultaneously utilizes both cathode and substrate as electron donors through direct electron transfer. In a cathode compartment poised at $+0.045 \mathrm{~V}$ vs. SHE, a metabolic shift in $C$. pasteurianum occurs toward NADH-consuming metabolite production such as butanol from glucose (20\% shift in terms of NADH consumption) and 1,3-propandiol from glycerol (21\% shift in terms of NADH consumption). Notably, a small amount of electron uptake significantly induces $\mathrm{NADH}$-consuming pathways over the stoichiometric contribution of the electrons as reducing equivalents. Our results demonstrate a previously unknown electroactivity and metabolic shift in the biochemical-producing heterotroph, opening up the possibility of efficient and enhanced production of electron-dense metabolites using electricity.

M icroorganisms have diverse capabilities in their use of various forms of electron acceptors $\left(\mathrm{O}_{2}\right.$, nitrate, sulfate, ferric iron $\left[\mathrm{Fe}^{3+}\right]$ oxide, heavy metals) as well as of electron donors (organic compounds, ferrous iron $\left[\mathrm{Fe}^{2+}\right]$ oxide, $\mathrm{H}_{2} \mathrm{~S}, \mathrm{H}_{2}$ ) to "make a living" in their natural habitats. In the past several decades, bioelectrochemical system (BES) using solid state electrodes as electron acceptors (at an anode) or electron donors (at a cathode) has emerged as a promising process to expand microbial metabolism to catalyze electrochemical redox reactions ${ }^{1,2}$. Although research on BES has mainly focused on electricity generation from the anode (e.g., microbial fuel cell), with the discovery and characterization of electron-donating microorganisms capable of direct electron transfer (e.g., Shewanella oneidensis, Geobacter sulfurreducens), microbial reductive reactions utilizing cathode-derived electrons as a reducing equivalent have recently received great attention in light of their ability to capture electricity from renewable solar and wind energies in the forms of fuels and chemicals ${ }^{2,3}$.

In $\mathrm{BES}$, electrons from the cathode can be supplied as the sole reducing equivalent for microbial $\mathrm{CO}_{2}$ reduction via direct electron transfer. Recent studies have shown that pure cultures of acetogenic microorganisms can produce organic compounds such as acetate, 2-oxobutyrate, and formate from $\mathrm{CO}_{2}$ through microbial electrosynthesis ${ }^{4,5}$. However, because of high electron demand for reduction of $\mathrm{CO}_{2}$, concentrations of microbial synthesis products are very low.

Besides electricity-driven microbial $\mathrm{CO}_{2}$ reduction, electrofermentation has been considered as an alternative electron-utilizing process that can overcome the limitations of bioelectrochemical $\mathrm{CO}_{2}$ reduction ${ }^{2,3,6}$. Electrofermentation is a hybrid metabolism in which electrons from the cathode are utilized as co-reducing equivalents in addition to carbon source-derived reducing equivalents (e.g., NADH) during heterotrophic fermentation, resulting in a higher production of electron-dense fuels and chemicals such as propionate, ${ }^{7}$ glutamic acid $^{8}$, butyric acid ${ }^{9}$, and butanol ${ }^{10}$. However, previous electrofermentation results were obtained with soluble exogenous electron mediators because the employed heterotrophic microorganisms were not electroactive. To accomplish electrofermentation, it is essential to find microorganisms that can take electrons from a cathode without exogenous mediators during heterotrophic fermentation. However, there has as yet been no report on electrofermentation-suitable microorganisms because heterotrophic microbes, even if they have the capability of direct electron transfer, likely prefer a soluble carbon source to a solid cathode as an electron donor. Specific 
microbial communities supplemented with acetate ${ }^{11}$ and glycerol ${ }^{12}$ have been found to be able to accept electrons from the cathode without exogenous mediators; however, the microbes responsible for electron uptake from the cathode, the interspecies interaction in the microbial community, and the possibility of hydrogen mediated electron transfer need to be identified ${ }^{11,12}$.

In the meantime, we have paid special attention to the metabolism of Clostridium pasteurianum DSM 525, which is different from those of other solventogenic clostridia: 1) it mainly produces butyrate and acetate from glucose, with a small amount of butanol ${ }^{13}$; 2) it can utilize glycerol as the sole carbon source and mainly produces more reduced compounds (butanol and 1,3-propanediol) compared to glucose-derived products (butyrate and acetate) (Supplementary Table 1, Supplementary Fig. 1); 3) metabolic shift from acetogenesis to solventogenesis is not shown at low $\mathrm{pHs}^{14}$. Given that the main products of $C$. pasteurianum are significantly dependent on the reduced level of the substrate, we chose C. pasteurianum as a good candidate bacterium for investigation of metabolic responses to cathodic electrons. The distinct bio-electrochemical characteristics of $C$. pasteurianum in nitrogen fixation ${ }^{15}$ and uranium reduction ${ }^{16}$ were also found to be attractive.

Here, we report that C. pasteurianum DSM 525, a known Grampositive bacterium, is capable of taking electrons directly from the cathode during fermentation even in the presence of electron-rich glucose and glycerol. Notably, the production of net NADH-consuming products such as butanol in glucose fermentation and, more notably, 1,3-propanediol (1,3-PD) in glycerol fermentation (Supplementary Table 1, Supplementary Fig. 1) were significantly enhanced by electron-accepting C. pasteurianum DSM525 even with a small amount of electron supply. Analysis of electron and NADH flows from dual electron donors (glucose or glycerol as soluble electron donor and the cathode as solid electron donor) to final products clearly revealed an electricity-driven metabolic shift to the reduction pathways in C. pasteurianum under BES, providing great opportunities to realize electrofermentation for production of biofuels and chemicals using sustainable electricity.

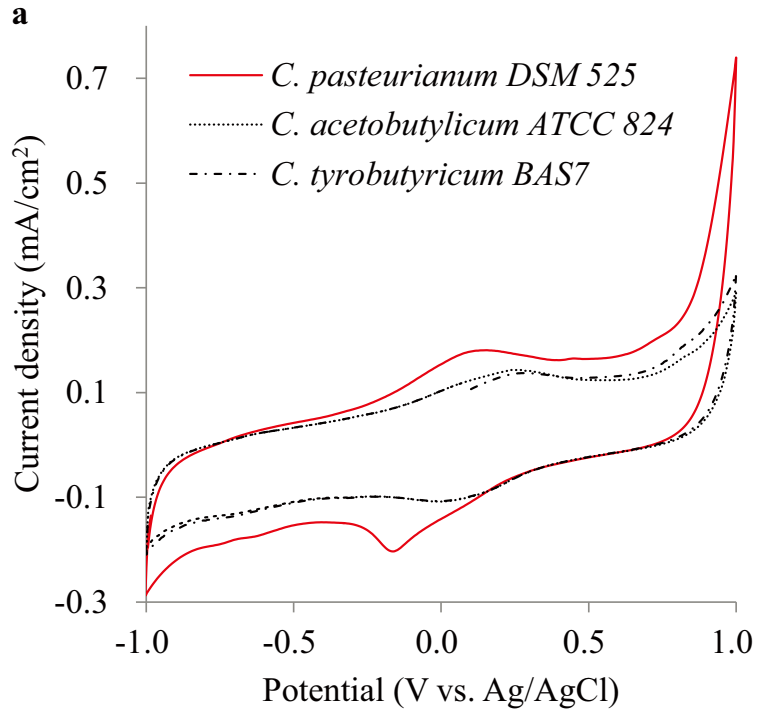

\section{Results}

C. pasteurianum accepts electrons from a cathode through direct electron transfer. We tested the electroactivity of $C$. pasteurianum DSM 525 in comparison with that of $C$. tyrobutyricum BAS7 and $C$. acetobutylicum ATCC 824, both of which were previously used for mediated-electrofermentation ${ }^{9,17}$. Among the tested Clostridium strains suspended for cyclic voltammetry (CV) analysis using a glassy carbon electrode, C. pasteurianum DSM 525 showed a significant electroactivity with definite redox peaks (Fig. 1a), while C. tyrobutyricum and C. acetobutylicum showed very weak and broad redox peaks. The shape of the reduction peak of $C$. pasteurianum (at $-0.16 \mathrm{~V}$ vs. $\mathrm{Ag} / \mathrm{AgCl}$ or $+0.045 \mathrm{~V}$ vs. $\mathrm{SHE}$ ) was different from that of the oxidation peak (at $0.125 \mathrm{~V}$ vs. $\mathrm{Ag} / \mathrm{AgCl}$ ), indicating that the redox reaction of suspended C. pasteurianum DSM 525 is quasi-reversible. Similar asymmetry of reduction peak and oxidation peak has been shown with Fe (III)-reducing and current-producing electroactive microorganisms ${ }^{18,19}$.

When three Clostridium strains were cultivated in the cathode compartment with a graphite felt electrode poised at $+0.045 \mathrm{~V}$ vs. SHE, only the $C$. pasteurianum DSM 525 culture showed current consumption $(0.5 \sim 1.5 \mathrm{~mA})$ during 48 hours of cultivation. As can be seen in Fig. 1b, the reduction peak of $C$. pasteurianum DSM 525 in the cathode compartment shifted to $-0.23 \mathrm{~V}$ vs. $\mathrm{Ag} / \mathrm{AgCl}(-0.025 \mathrm{~V}$ vs. SHE) and became broader, probably due to the rough and porous surface structure of the graphite felt ${ }^{20}$. No redox reactions were found when CV was analyzed with fresh medium and cell-free culture supernatant (spent medium) using a sterilized graphite felt electrode (Fig. 1b), implying the absence of electroactive components in the supernatant. For further analysis, after the biofilm was formed on the cathode (graphite felt) during 40 hours of cultivation in BES $(+0.045 \mathrm{~V}$ vs. SHE), the suspended culture, including free-swimming cells, was drained out and fresh medium was re-filled in the cathode compartment under anaerobic conditions by purging the compartment with argon gas. When the potential (+0.045 V vs. SHE) was applied again, the current consumption immediately recovered to $1.2 \mathrm{~mA}$; it further increased as glucose consumption and cell growth resumed (Supplementary Fig. 2), indi-

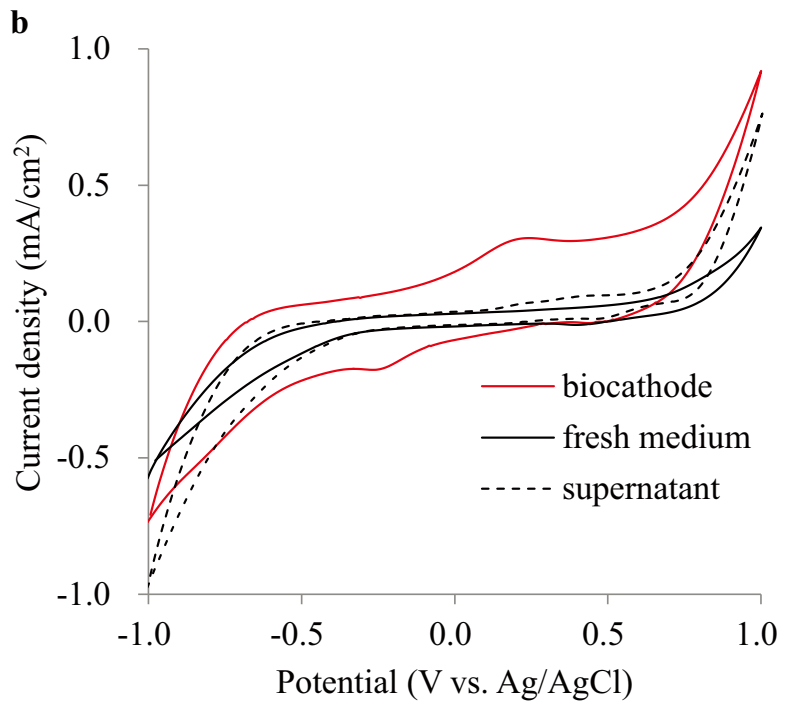

Figure $1 \mid$ The cyclic voltammetry (CV) study. (a) The CV of various Clostridium strains. The Ag/AgCl electrode was used as the reference electrode and a glassy carbon electrode as the working electrode. The scan rate was $50 \mathrm{mV} / \mathrm{s}$. Each Clostridium strain was cultured in MP2 medium (glucose $100 \mathrm{mM}$ ) and $\mathrm{CV}$ was analyzed in a jar separately. Only in C. pasteurianum, the reduction peak was shown at $-0.16 \mathrm{Vvs}$. $\mathrm{Ag} / \mathrm{AgCl}(+0.045 \mathrm{Vvs}$. SHE). (b) The CV of $C$. pasteurianum cells (biofilm on the cathode and suspended culture) cultivated for 40 hours in the cathode compartment under the potential of $-0.16 \mathrm{~V}$ vs. $\mathrm{Ag} / \mathrm{AgCl}(+0.045 \mathrm{~V}$ vs. SHE). The scanning rate was $50 \mathrm{mV} / \mathrm{s}$ and $\mathrm{Ag} / \mathrm{AgCl}$ was used as the reference electrode. The biocathode was the working electrode. 
cating electron transfer without suspended mediators between the cathode and the biofilm of C. pasteurianum DSM 525. S. oneidensis ${ }^{21}$ and Thermincola ferriacetica ${ }^{22}$ generating current by direct electron transfer also showed similar electroactivity patterns when the bioanode was tested in a microbial fuel cell system.

Confocal fluorescence images (Supplementary Fig. 3) from a biocathode stained with live/dead staining also supported the idea of a direct electron transfer through active biofilm formation. Confocal fluorescence images showed an increase of cell attachment on the graphite felt (Supplementary Fig. 3a) compared to cell attachment on the control (immersed graphite felt without electricity, Supplementary Fig. 3b). As can be seen in Supplementary Fig. 3a, live cell attachment along the configuration of the graphite fibers in the cathode was found in BES. The cathode and the cell surface have negative charge. Due to electrostatic repulsive interaction, bacterial adhesion on the cathode is likely unfavorable. However, live cell attachment on the cathode and active biofilm formation were clearly shown (Supplementary Fig 3a). Therefore, it is suggested that $C$. pasteurianum actively interacted with the cathode against the electrostatic repulsion in BES, accepting electrons even in the presence of a soluble electro-rich carbon source, glucose.

BES changes extracellular structures and electronegativity of $C$. pasteurianum. Biofilm cells of $C$. pasteurianum on the cathode poised at $+0.045 \mathrm{~V}$ vs. SHE were observed through SEM analysis; it was found that C. pasteurianum DSM 525 cells stacked with each other on the cathode (Supplementary Fig. 4). Extracellular appendages were shown only in the biocathodes of BES, not in the graphite felt of the control culture (open circuit). Although the role of the extracellular appendages observed in the bio-cathode cannot be elucidated from SEM images alone, BES was likely to cause significant changes in the extracellular structure of C. pasteurianum DSM 525 attached on the cathode.

In addition to SEM micrographs, TEM images were analyzed with cells detached from biofilm formed with electricity (posed at $+0.045 \mathrm{~V}$ vs. SHE) (Fig. 2a) and without electricity (Fig. 2b). Interestingly, tiny graphite felt pieces were found to surround the cells grown with electricity (Fig. 2a), while cells collected under open circuit conditions appeared to repel graphite felt pieces (Fig. 2b), suggesting that there was the change of electrostatic interaction between cells and the graphite felt in BES. For further investigation, the zeta potential of cells grown with and without electricity was measured. The surface of cells under an open circuit was electronegative, with a zeta potential of $-16.5 \pm 4.4 \mathrm{mV}$, whereas the zeta potential of cells in BES was near zero $(-4.5 \pm 3.8 \mathrm{mV})$ implying an almost electro neutral state. The surface charge of a bacterial cell is known to be an important factor at the initial attachment step to the solid surface through electrostatic interactions ${ }^{23}$. Considering that the cathode in BES was negatively charged, the surface charges of
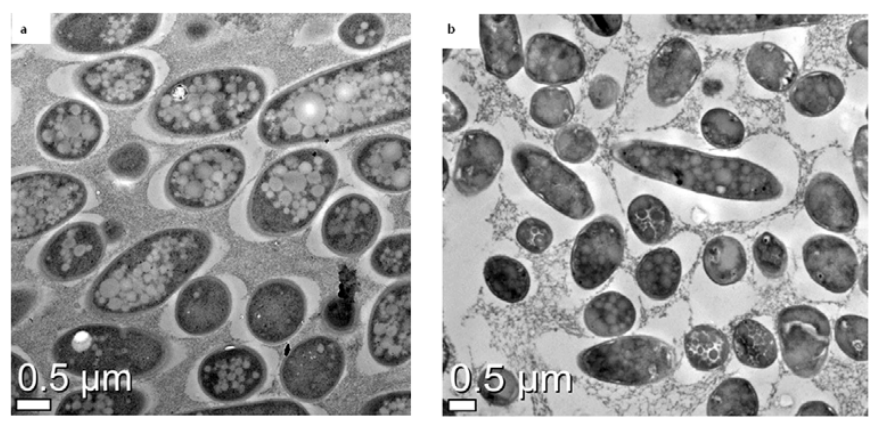

Figure $2 \mid$ TEM images of cells collected from the graphite felt (cathode) (a) poised at $+0.045 \mathrm{~V} v$ s. SHE and (b) under open circuit (no electricity). The phase bright granulose within the cell in is clostridial form responsible for solvent production. the electroactive cells were likely modified to be less electronegative so that the cells could more easily attach to the cathode and consume currents. It should be noted that the opposite tendency of electronegativity for current-producing microorganisms has been found: $G$. sulfurreducens and Shewanella species were reported to be electronegative on the basis of zeta potential ${ }^{24}$, which would explain the attachment of Geobacter and Shewanella cells on positively charged anode surfaces. Because Gram-positive bacteria do not possess an outer membrane, the composition of peptidoglycan or extracellular polymeric substances might be altered in BES to change the electronegativity of the cell surface. Further characterization of $C$. pasteurianum DSM 525 cells in BES would provide insight elucidating the interaction between cathode and cells for direct electron transfer.

Electron uptake by $C$. pasteurianum increases butanol production from glucose. As C. pasteurianum accepted electrons from a poised electrode, we hypothesized that microbial electron uptake might induce the production of more reduced products from glucose. To check the change in fermentation behavior, cell growth and product concentrations were analyzed in both control (without electricity) (Fig. 3a) and electrochemically driven system (Fig. 3b).

Unexpectedly, lactate was produced in the exponential phase at the expense of acetate and butyrate only under BES. Butanol production started to increase when lactate uptake was initiated (Fig. 3b). Interestingly, the increase of butanol production (10.1 mM) after 20 hours of cultivation was similar to a half of the consumed lactate (18.3 mM). Because 2 moles of lactate can be stoichiometrically converted to $1 \mathrm{~mol}$ of butanol through microbial metabolism, most of the increased butanol production in BES was likely attributed to lactate consumption. In order to investigate whether lactate triggered butanol production, different concentrations of lactate were added without electricity to liquid cultures of $C$. pasteurianum DSM 525 growing on glucose. Unlike the case of BES, the increase of butyrate production was much higher than that of butanol production (see Supplementary Fig. 5). That is, conversion of lactate to butyrate is a favorable metabolic pathway in the absence of electricity, whereas the conversion of lactate to butanol apparently occurred in BES. Considering all points together, it can be suggested that lactate detected in BES likely plays an important role as an intermediate metabolite, consuming electricity-derived reducing power. Electron uptake in BES might induce NADH generation and increase lactate formation, which is the first and simplest pathway to consume NADH after glycolysis (1 mol NADH consumption per 1 mol lactate production from pyruvate by lactate dehydrogenase) (Supplementary Fig. 1). Then, increased lactate concentration and electricity might drive metabolic pathways to convert lactate to butanol (net NADH-consuming product in glucose fermentation) for further consumption of NADH ( 2 mol NADH consumption per 1 mol butanol production from 2 mol lactate).

To investigate whether electricity increased the intracellular reducing power, the $\mathrm{NADH} / \mathrm{NAD}^{+}$ratios of the cells in the control and BES were analyzed. As can be seen in Fig. 3c, the intracellular NADH level (per $\mathrm{NAD}^{+}$) increased to $\sim 1$ and then decreased to 0.2 during butanol production in BES. On the other hand, the ratio of NADH/ $\mathrm{NAD}^{+}$in the control was relatively balanced, with only small variation (Fig. 3c). NADH/NAD ${ }^{+}$is usually balanced through $\mathrm{NADH}$ production in glycolysis ( $2 \mathrm{NADH}$ production during 2 pyruvate formation from glucose) and NADH consumption in the acid and alcohol production pathways $\left(\mathrm{NAD}^{+}\right.$regeneration) as shown in the control. However, in BES (Fig. 3c), the NADH/NAD ${ }^{+}$ratio was not balanced until butanol production occurred. This result indicates that electrons from the cathode affected the intracellular redox conditions, possibly by reducing $\mathrm{NAD}^{+}$to $\mathrm{NADH}$ in the cells. Lactate production followed by butanol production seemed a metabolic response to consume more NADH by producing more reduced compounds (Supplementary Table 1). 
a

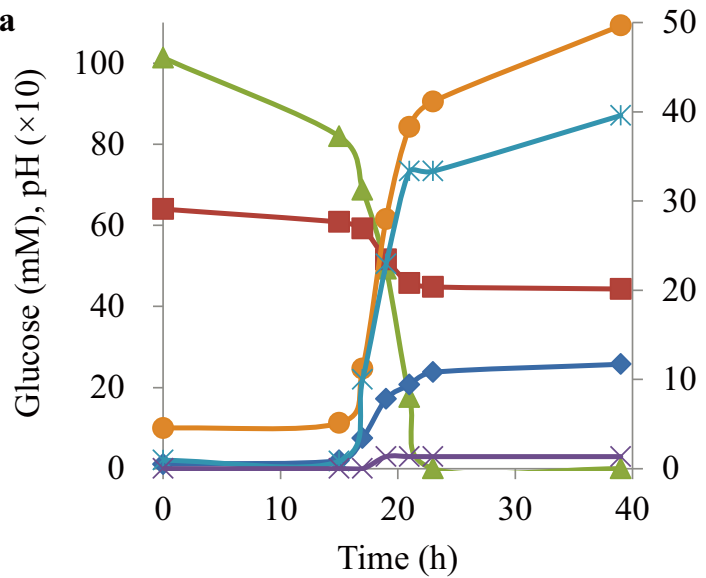

50

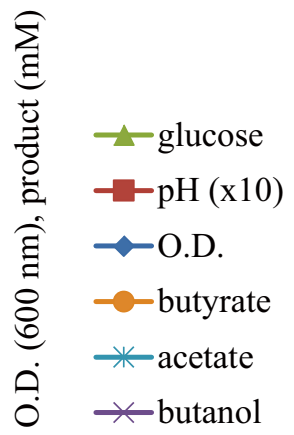

b

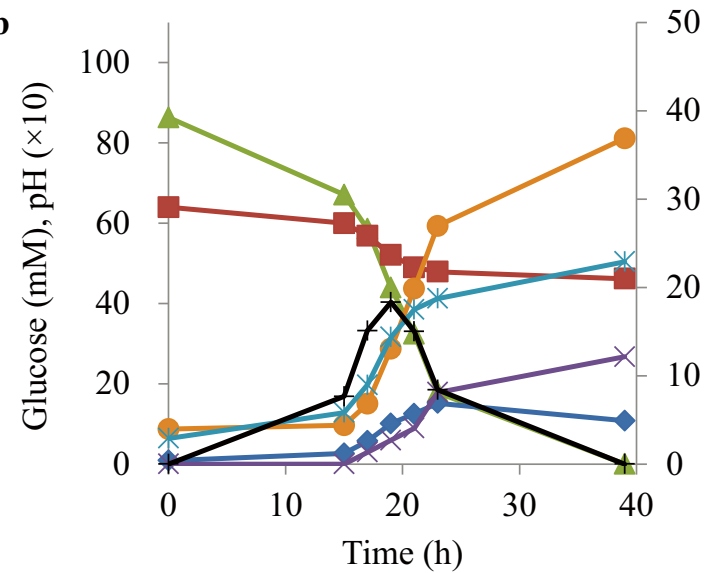

50

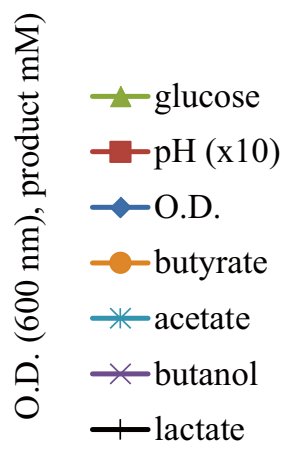

c

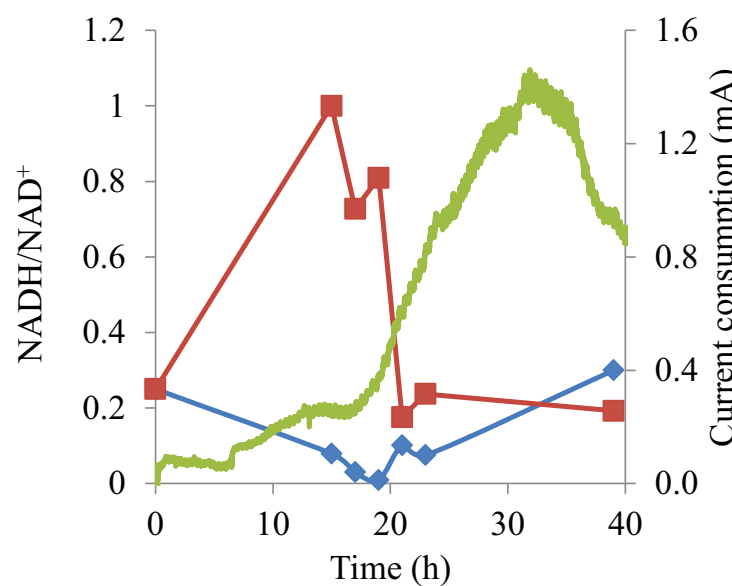

$\neg \mathrm{NADH} / \mathrm{NAD}+($ control)

$-\mathrm{NADH} / \mathrm{NAD}+(\mathrm{BES})$

Current consumption

Figure $3 \mid$ The time profile of glucose fermentation by $C$. pasteurianum (a) in control (without electricity) and (b) in bioelectrochemical system poised at $+0.045 \mathrm{~V}$ vs. SHE. It shows one representative result out of 11 replicate experiments. (c) The current consumption increased with microbial growth but decreased at the end of stationary phase with cell lysis (surface area of electrode: $108 \mathrm{~cm}^{2}$ ). The ratio of intracellular NADH/NAD in bioelectrochemical system and control (without electricity). Electrochemically introduced electrons increased NADH/NAD ${ }^{+}$ratio and decreased with butanol formation.

Overall, as can be seen in Fig. 3, butanol production increased (2.1 vs. $12.2 \mathrm{mM}$ ) and acid production decreased (49.7 vs. $36.9 \mathrm{mM}$ in butyric acid; 39.6 vs. $22.9 \mathrm{mM}$ in acetic acid) in the presence of electricity. The $\mathrm{pH}$ change in the control was not much different from that in BES, whereas O.D. decreased suddenly during the stationary phase in BES. Cell disruption has been reported in Enterobacter exposed to weak electricity $(10 \mathrm{~mA})^{25}$ and in C. tyrobutyricum under mediated BES 9 . There was no significant difference in gas composition between the control and BES $\left(\mathrm{H}_{2} /\right.$
$\mathrm{CO}_{2}=1.09$ in the control and 1.10 in BES), indicating little concern on electrochemical hydrogen production. The potential applied in this study $(+0.045 \mathrm{~V}$ vs. SHE) was far higher than the electrochemical hydrogen production potential $(-0.41 \sim$ $-0.27 \mathrm{~V}$ vs. SHE at $\mathrm{pH}$ range $4.5 \sim 7.0$ ), which also supports little abiotic hydrogen production in this BES system. This is different from the previously reported BES system in which electrochemically-produced hydrogen at the potential of $-0.9 \mathrm{~V}$ vs. SHE could mediate electron transfer in microbial communities ${ }^{11,12}$. 
Table 1 The comparison of growth rate, substrate uptake, and productivity in two conditions. The control was the fermentation of glucose by $C$. pasteurianum in MP2 medium and BES indicated the bioelectrochemical system with the same medium but poised at $+0.045 \mathrm{~V}$ vs. SHE. The value is average and one standard deviation (in parentheses) of 11 replicates

\begin{tabular}{lcc} 
& Control & BES \\
\hline Maximum O.D. $(600 \mathrm{~nm})$ & $10.6( \pm 0.9)$ & $6.2( \pm 1.1)$ \\
Growth rate $\left(\mathrm{h}^{-1}\right)$ & $0.41( \pm 0.05)$ & $0.25( \pm 0)$ \\
Productivity $(\mathrm{mM} / \mathrm{h})$ & $10.4( \pm 0.3)$ & $8.2( \pm 2.7)$ \\
Glucose uptake rate & $7.0( \pm 0.2)$ & $3.7( \pm 0.5)$ \\
Butyrate productivity & $6.5( \pm 0.8)$ & $3.2( \pm 1.7)$ \\
Acetate productivity & $0.5( \pm 0.3)$ & $1.2( \pm 0.1)$ \\
Butanol productivity & \\
Final concentration $(\mathrm{mM})$ & & \\
Butyrate & $51.1( \pm 5.7)$ & $43.1( \pm 6.8)$ \\
Acetate & $38.3( \pm 5.0)$ & $33.3( \pm 11.7)$ \\
Butanol & $5.4( \pm 4.1)$ & $13.5( \pm 2.7)$ \\
\hline
\end{tabular}

The growth and fermentation patterns of C. pasteurianum with and without electricity from 11 replicated experiments are summarized in Table 1. In BES, the growth rate and the maximum O.D. were significantly lower than those in the control. The formation of biofilm on the cathode in BES (Supplementary Fig. 3a) may be a possible answer for the low $\mathrm{OD}_{600}$ values of suspended cultures. The productivities of acids decreased to approximately half of those in the control; the butanol productivity increased over 2 -fold (Table 1). This suggests that the electron uptake by C. pasteurianum in BES resulted in a metabolic shift to quickly consume the reducing equivalent (NADH) by producing butanol rather than acids. To investigate how electron and carbon flows were changed in electrofermentation, the electron and carbon balance was set up with final products (Supplementary Table 2). Electron equivalent distributions in the control and BES were analyzed on the basis of half reaction (Supplementary Table 3). There were $14 \sim 20 \%$ of electron and carbon loss in balance possibly because of gas leaking and omitting biofilm biomass in calculation. Interestingly, although the electrons from the cathode in BES was only $0.2 \%$ of the total electron supply, the electron distribution of butanol significantly increased in electrofermentation (16\%) compared to the control (5\%). This result indicates that electron flow to butanol significantly increased during electrofermentation even with small electricity.

\section{C. pasteurianum shows current consumption-dependent final} product distribution in glycerol fermentation. Given that current consumption in a range of $0.5 \sim 1.5 \mathrm{~mA}$ resulted in a more than 2 fold increase of butanol production, we investigated whether butanol production could be increased further by stimulating more current consumption with more biofilm on the cathode surface. To prepare mature biofilm on the electrode surface, the suspended culture in the cathode was replaced with a fresh medium under anaerobic condition while the cathode with the biofilm remained in the cathode compartment. When this process was repeated 3 times in 24 hours, the current consumption increased to $5 \mathrm{~mA}$ (Supplementary Table 4), but no more increase of current consumption was observed despite repeated batches. The final product distribution changed with the repeating number of batches; however, butanol yield did not increase further even when current consumption was increased more than 3 -fold $(1.5 \mathrm{~mA}$ to $5 \mathrm{~mA})$. Instead, in the $3^{\text {rd }}$ batch, ethanol and butyrate concentration increased, rather than that of butanol (Supplementary Table 4). This result suggests that the reducing power, derived from electrons from the cathode, stimulated butanol production at first; however, butanol production did not increase with current consumption in the $2^{\text {nd }}$ and $3^{\text {rd }}$ batches, probably because of an intrinsic metabolic limitation of $C$. pasteurianum toward butanol production in glucose fermentation. Instead, ethanol and butyric acid production might be stimulated in order to consume reducing equivalents.

Unlike other butanol-producing clostridia species, C. pasteurianum is able to use glycerol as a sole carbon source and produce butanol and 1,3-PD as main products (Fig. $4 \mathrm{a})^{26}$. Considering that glycerol ( $4.7 \mathrm{e}^{-}$equivalent/C-glycerol) is a more reduced carbon source than glucose is ( $4 \mathrm{e}^{-}$equivalent/C-glucose), C. pasteurianum in glycerol fermentation is capable of efficiently balancing the intracellular NADH/NAD ${ }^{+}$ratio by actively producing reduced metabolites such as 1,3-PD (net NADH-consuming, Supplementary Table 1) and butanol (net NADH-neutral in glycerol fermentation). This is different from glucose fermentation, in which the favorable metabolic pathway is butyric acid production (net NADH-neutral), not butanol production (net NADH-consuming). To investigate whether an active NADH-consuming metabolism during glycerol fermentation would affect current consumption and product profiles, the BES batch, supplemented with glycerol as a carbon source, was repeated with the remaining bioelectrode in the cathode compartment. Glycerol $(250 \sim 300 \mathrm{mM})$ was completely consumed after 48 hours of cultivation during the repeated batches and electron consumption increased to $9 \mathrm{~mA}$ in the $3^{\text {rd }} \sim 4^{\text {th }}$ batches, which level was higher than that with repeated BES batches with glucose $(5 \mathrm{~mA}$, Supplementary Table 4). Unlike glucose fermentation, glycerol fermentation led to no lactate production (Fig. 4b) because lactate is net NADH-generating product in glycerol fermentation (Supplementary Table 1). Of interest was that a higher current consumption resulted in more 1,3-PD production and a shift of the major product from butanol to 1,3-PD (Fig. 4c). This result implies that more current consumption could induce the metabolic pathway to produce a more net $\mathrm{NADH}$-consuming compound $(1,3-\mathrm{PD})$ in glycerol fermentation (Supplementary Table 1). Glycerol reduction to 1,3-PD occurs through only two-step NADH-consuming enzymatic reactions and glycerol reduction is independent of NADH generation pathway (i.e., glycerol oxidation pathway to pyruvate) (Supplementary Fig. 1). That is, at a higher current consumption, $\mathrm{NADH}$ can be quickly consumed in a fast and independent way by producing 1,3-PD. On the contrary, in glucose fermentation, butanol production from pyruvate (NADH consumption pathway) is always coupled with glucose oxidation to pyruvate (NADH generation pathway) and occurs through multiple metabolic pathways (Supplementary Fig. 1). This lack of a simple and independent net NADH-consuming pathway in glucose fermentation might limit current consumption and more reduced compound production. Overall, as clearly shown in Fig. 4c, final product distribution was current consumption-dependent in glycerol fermentation, supporting the idea that electricity-driven metabolic pathway shift occurred in C. pasteurianum under BES to produce more reduced compounds (net NADH-consuming compounds).

Small amount of electron uptake significantly stimulates NADHconsuming metabolism. Because cathodic electrons are supposed to be utilized as co-reducing equivalents and consequently are supposed to affect metabolic electron flow in electrofermentation, we compared reducing equivalent (NADH and cathodic electrons) flow from electron donors (carbon source and cathode) to final products in the control and in BES; we investigated how cathodic electrons affected intracellular redox reactions. According to Equation (1) (refer to Methods section), the electron consumption in BES was $1.51 \mathrm{e}^{-}$mmol (equivalent to $0.8 \mathrm{mmol} \mathrm{NADH}$ ) during glucose electrofermentation. Surprisingly, this electricity-derived NADH generation was only $1.5 \%$ of glucose-derived NADH generation (Fig. 5a, Supplementary Table 5). In glycerol fermentation, the electricity-derived $\mathrm{NADH}$, theoretically calculated from the electron consumption (4.96 $\mathrm{e}^{-}$mmol from equation 1), was also only $2.3 \%$ of the glycerol-derived NADH (Fig. 5c, Supplementary Table 6). This 
a

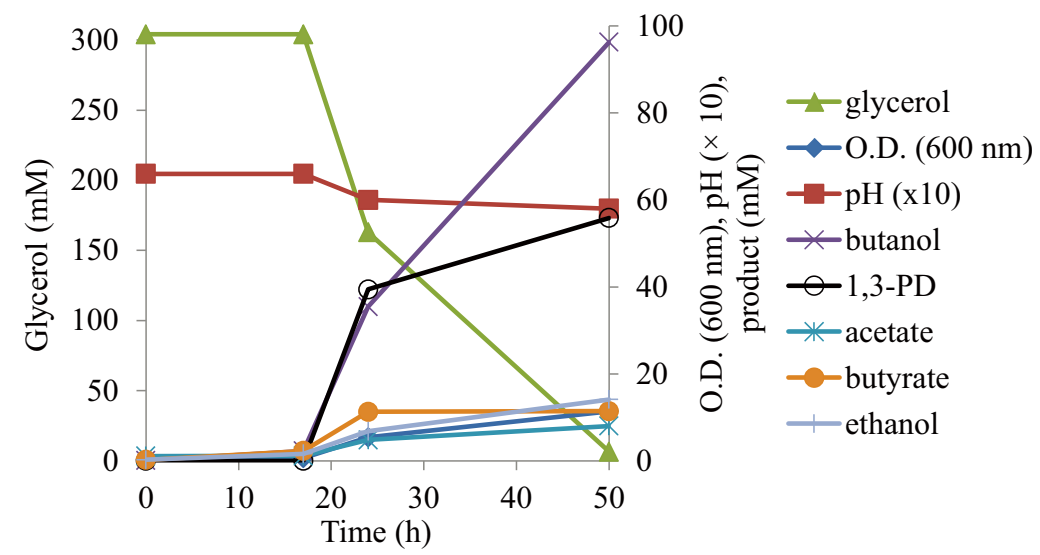

b

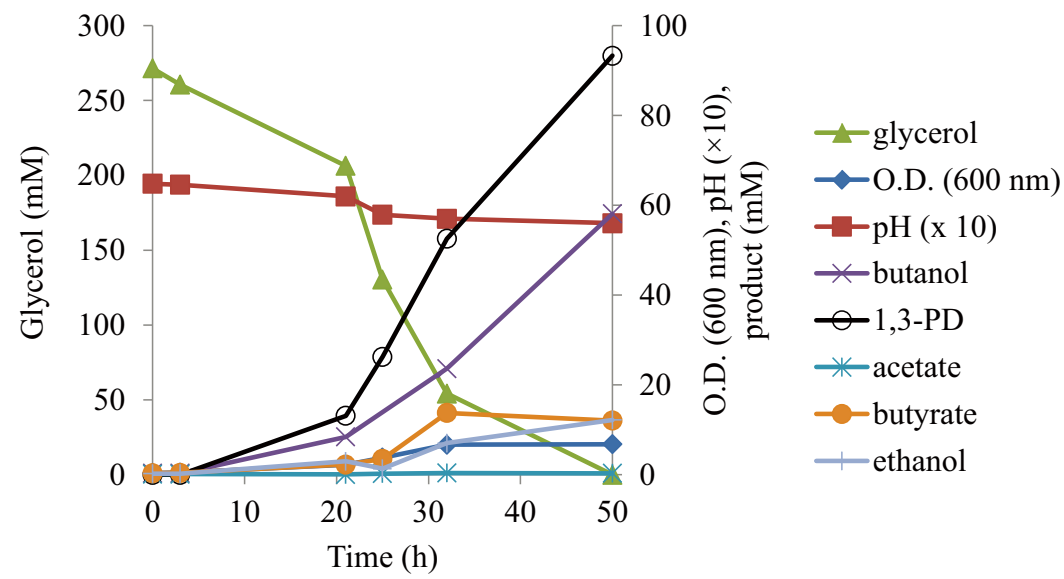

c

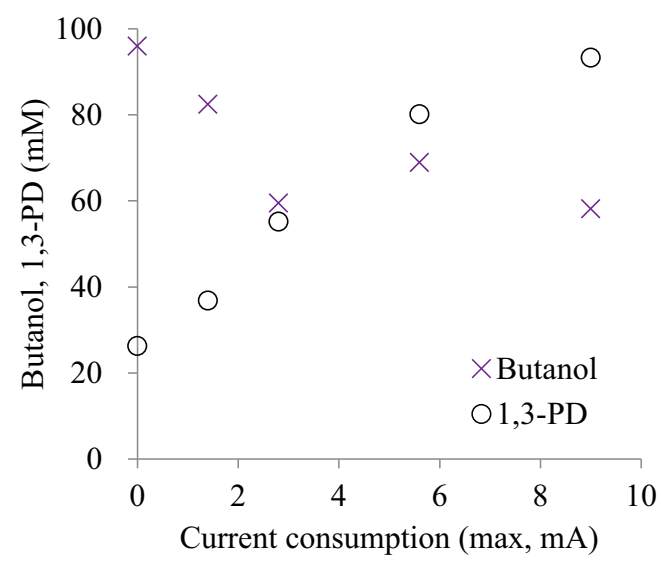

Figure $4 \mid$ The glycerol fermentation by $C$. pasteurianum (a) in control (without electricity) and (b) in bioelectrochemical system poised at $+0.045 \mathrm{~V}$ vs. SHE with $9 \mathrm{~mA}$ current consumption (c) The current consumption-dependent main product shift from butanol to 1,3-PD in glycerol electrofermentation (surface area of electrode: $108 \mathrm{~cm}^{2}$ ). The current consumption was recorded at the stationary phase with the maximum value and the concentration of product was final concentration when C. pasteurianum completely consumed supplied glycerol.

indicates that NADH generation from glucose and glycerol accounted for most of $\mathrm{NADH}$ in BES; however, even a small amount of electricity significantly changed the metabolism toward net NADHconsuming compounds. This was more clearly shown when we compared how NADH derived from carbon sources and electrons was consumed in the control and in BES. As can be seen in Fig. 5b and Fig. 5d, the percentage of NADH consumption (Equation 2 in Methods, Supplementary Table 5 and 6) out of total NADH generation (Equation 3 in Methods, Supplementary Table 5 and 6) significantly increased from $61 \%$ to $80 \%$ in the glucose electrofermentation and from $78 \%$ to $96 \%$ in the glycerol fermentation. Interestingly, $\mathrm{NADH}$ consumption in BES increased more than the amount of electron-derived NADH (Fig. $5 \mathrm{a}$ vs. $5 \mathrm{~b}$; Fig. $5 \mathrm{c}$ vs. Fig. 5 d). Because the amount of electricity-derived NADH was far smaller that of the substrate-derived NADH (Fig. 5a, Fig. 5c), a higher portion of NADH consumption out of NADH generation in BES (Fig. 5b, Fig. 5d) is likely attributed to not only the utilization of cathodic electrons as additional reducing equivalents but also to the significant stimulation of carbon source-derived NADH consumption toward the production of more reduced compounds by cathodic electrons.

The increase of NADH consumption toward net NADH-consuming compound production ( 11 vs. $31 \%$ for butanol in Fig. $5 \mathrm{~b}$ and 5 vs. $26 \%$ for $1,3-P D$ in Fig. 5 d) mainly contributed to the increased 

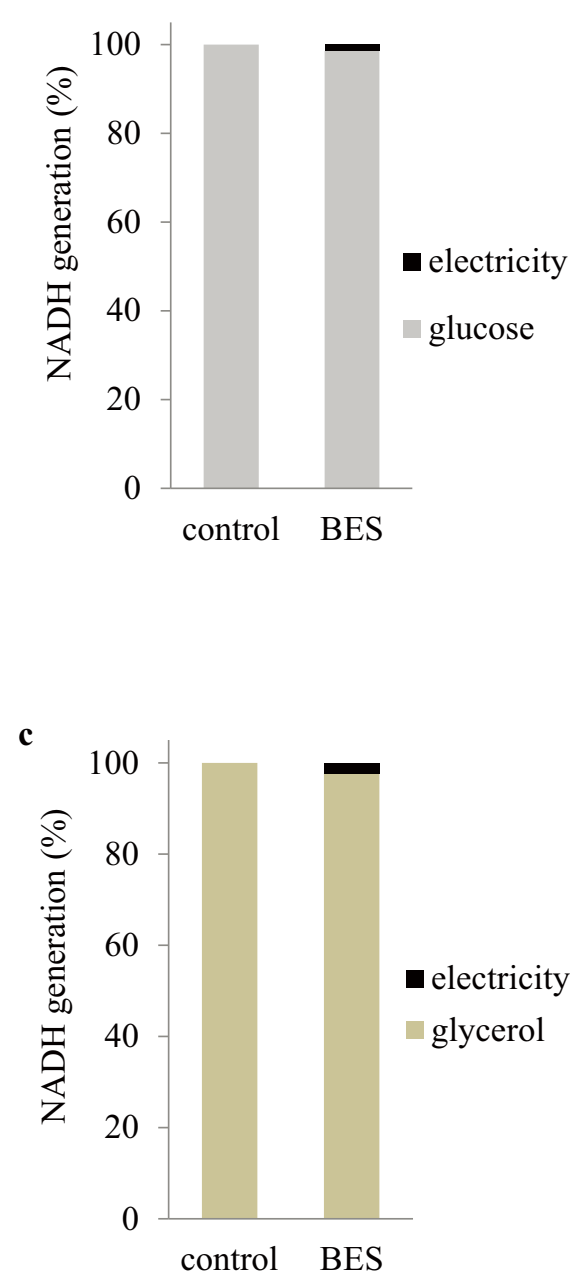

b

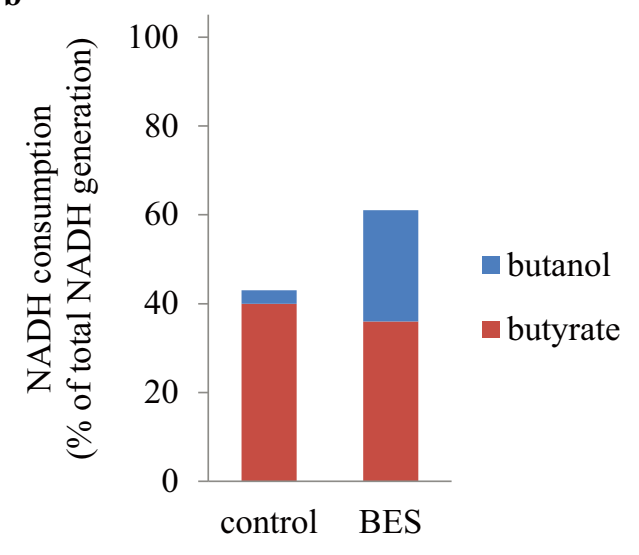

d

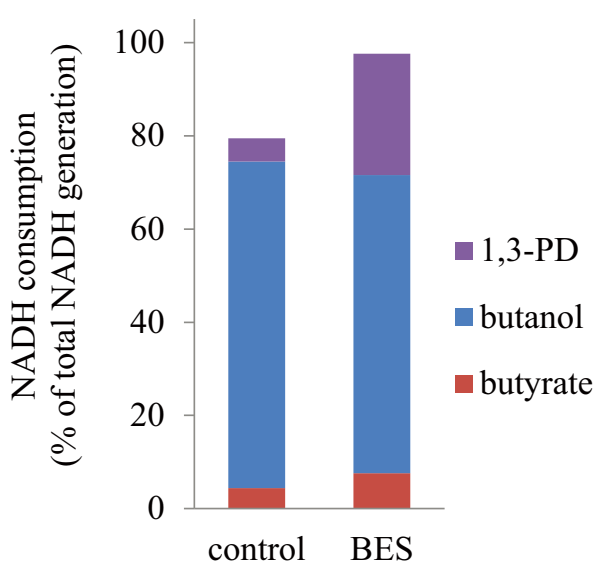

Figure 5 NADH flows in the control and BES. Values for glucose fermentation (a,b) were calculated from the average value of 11 replicates shown in Table 1. For the calculation of values in BES with glycerol (c,d), the data obtained at $9 \mathrm{~mA}$ of current consumption (Fig. $4 \mathrm{~b}$ ) were used. (a) NADH generation (\%) and (b) NADH consumption (\%) in glucose fermentation were determined with the values in Supplementary Table 5. (c) NADH generation (\%) and (d) NADH consumption (\%) in glycerol fermentation were determined with the values in Supplementary Table 6.

percentage of total $\mathrm{NADH}$ consumption in $\mathrm{BES}$, indicating that metabolic shift to reduction pathways was predominant even with a small amount of electrons. Considering that only $0.66 \mathrm{mM}$ of butanol and $8.3 \mathrm{mM}$ of 1,3-PD can be stoichiometrically produced by utilizing electricity-derived $\mathrm{NADH}$ in glucose and glycerol electrofermentation, respectively, the dramatic metabolic shift in electron-accepting C. pasteurianum shown here (Fig. 3, Fig. 4, and Fig. 5) is much more than what we were expecting from electrofermentation, producing more reduced compounds in higher concentrations not only by utilizing electrons as a co-reducing equivalent but also by significantly inducing a reductive metabolism greater than that the electrons themselves can stoichiometrically induce.

\section{Discussion}

Here, we have shown a direct electron transfer from a cathode to $C$. pasteurianum and an electricity-derived reducing power induced $\mathrm{NADH}$-consuming metabolic pathway in glucose and glycerol fermentation. This is the first report to present a wild type of pure culture (C. pasteurianum) that not only accepts electrons directly from the cathode even in the presence of electron-rich glucose and glycerol (heterotrophic growth), but that also produces a higher amount of net NADH-consuming compounds from substrates, beyond that which supplemented electricity can theoretically achieve. The immediate current recovery in the $2^{\text {nd }}$ batch of BES
(Supplementary Fig. 2) supports direct electron transfer. In addition, considering that cell adhesion to insoluble electron acceptors (ferric iron oxide) is not required for Shewanella in the presence of soluble electron shuttles ${ }^{24}$, the biofilm of $C$. pasteurianum on the cathode also supports direct electron transfer from the cathode to the cells.

The mechanism of direct electron transfer in C. pasteurianum is as yet unknown. Gram-positive bacteria lack an outer membrane and it is structurally hard for them to carry electrons, whereas Gram-negative bacteria like Geobacteraceae have complicated electron transfer chains $^{27}$. Nonetheless, it was also reported that Gram-positive Clostridium strains like C. ljungdahlii and C. aceticum were able to consume electrons from the cathode to produce small amounts of multicarbon-compounds using $\mathrm{CO}_{2}$ as the sole carbon source. Our studies suggest that the surface interaction between electrode and microorganism could affect extracellular electron transfer and it may be future study to elucidate the mechanism of the direct electron transfer of C. pasteurianum.

Electrochemically introduced reducing power appeared to significantly affect intracellular redox conditions (Fig. 5) and consequently stimulate the production of net $\mathrm{NADH}$-consuming metabolites like butanol in glucose fermentation and, more notably, 1,3-PD in glycerol fermentation. In early studies, C. acetobutylicum increased butanol production up to $26 \%$ using electrically reduced methyl viologen as an electron mediator ${ }^{17}$. However, C. pasteurianum was 
able to directly accept electrons from the cathode without any mediators and butanol production increased more than 2-fold compared to the case of glucose fermentation without electricity. The metabolic shift to the net $\mathrm{NADH}$-consuming reaction in glycerol fermentation was clearly current consumption-dependent (Fig. 4c) and more significant than it was in glucose fermentation. This result is likely due to the presence of a simple and separate reduction pathway in the glycerol fermentation process (i.e., 1,3-PD production); this pathway likely responds to BES conditions actively by consuming reducing power. Lactate production as an intermediate from glucose electrofermentation also supports the idea of the importance of a relative simple pathway to efficiently consume NADH in BES.

Recently, the utilization of electrons from a cathode as a reducing equivalent in biofuel production has been studied with carbon dioxide as a carbon source; this process mimics natural photosynthesis in plants ${ }^{28-30}$. However, a much higher electron uptake is required for $\mathrm{CO}_{2}$ reduction compared to that necessary for microbial biochemical production from general carbon sources such as sugars and glycerol. This high demand for electrons and, as mentioned in the case of glucose electrofermentation, the lack of a relatively simple and active reduction pathway, might limit the production of electrofuel from $\mathrm{CO}_{2}$. Alternatively, co-utilizing organic substrates and electrons from a cathode as a reducing power through electrofermentation has been suggested as a way to overcome the limitations of $\mathrm{CO}_{2}$ reduction using electrons ${ }^{2,3}$; however, there have been no reports on single pure microorganism that can perform electrofermentation. It is of interest that $C$. pasteurianum actively attached to the cathode to uptake electrons even though there was abundant amount of electron-rich glucose. In the case of microbial fuel cell (which is an electron-producing process), G. metallireducens only donated electrons to an anode in the absence of soluble electron acceptors ${ }^{31,32}$. On the contrary, C. pasteurianum used dual electron donors, i.e., glucose or glycerol (soluble), and the cathode (insoluble). This characteristic of C. pasteurianum of simultaneously utilizing a reducing equivalent derived from the cathode and substrates will be very advantageous to facilitate electrofermentation for biofuel and biochemical production.

It is generally known that electroactive microorganisms do not possess biochemical production pathways and that biochemical-producing heterotrophic microorganisms are not electroactive ${ }^{33}$. Therefore, this unique characteristic of C. pasteurianum (an electroactive biochemical-producing heterotrophic microorganism) is extraordinary. It should be noted that the electroactivity of C. pasteurianum had not been discovered until we demonstrated it here, although C. pasteurianum is a well-known Gram-positive bacterium that has been studied for a long time by many researchers ${ }^{14-16,26,34}$. This indicates that, in addition to isolating novel electroactive microorganisms, exploring known microorganisms might yield unexpected opportunities to discover previously unknown electrochemical activity. The results presented here could be the next step toward unveiling a natural bio-electrochemical versatility, including such a versatility in heterotrophs. Further study on the mechanism of direct electron transfer and elucidation of the metabolic regulation in BES will provide valuable information to open the next door toward efficient biofuel and biochemical production using sustainable electricity.

\section{Methods}

Bacterial strains and medium. C. pasteurianum DSM 525 and C. acetobutylicum ATCC 824 were purchased from DSMZ (Braunschweig, Germany) and the American Type Culture Collection (ATCC, Rockville, MD), respectively. C. tyrobutyricum BAS7 was isolated as a butyric acid producer from sucrose ${ }^{9}$. The strains were precultured in argon gas-purged MP2 medium (adjusted pH 6.5 with $5 \mathrm{M} \mathrm{KOH})^{35}$, including $100 \mathrm{mM}$ glucose and $100 \mathrm{mM}$ MES (2-(N-morpholino) ethanesulfonic acid) at $37^{\circ} \mathrm{C}, 125 \mathrm{rpm}$. Bacteria were grown in $60-\mathrm{mL}$ serum bottles sealed with butyl rubber stoppers and an aluminum crimp containing $20 \mathrm{~mL}$ of medium under an argon gas phase (99.9\%). Overnight-cultivated Clostridium strains were inoculated (5\%) into a cathode compartment filled with $300 \mathrm{~mL}$ medium. Unless otherwise stated, all chemicals were purchased at the highest grade available and used without further purification.

Bioelectrochemical systems (BES). An H-type reactor with a dual-chamber was employed as the bioelectrochemical system. The volume of each chamber was $450 \mathrm{~mL}$; chambers were physically separated by a Nafion 117 cation-exchange membrane (Naracell-tech, South Korea). The cathode was a graphite felt electrode $(4.5 \mathrm{~cm} \times 12 \mathrm{~cm})$ and the anode was a Pt plate electrode $(4.5 \mathrm{~cm} \times 12 \mathrm{~cm})$. After the reactor was autoclaved, the anode compartment was filled with $0.2 \mathrm{M}$ hexacyanoferrate $\left[\mathrm{K}_{4}\left(\mathrm{Fe}(\mathrm{CN})_{6}\right) \cdot 3 \mathrm{H}_{2} \mathrm{O}\right]$ and the cathode compartment was filled with separately autoclaved medium $(300 \mathrm{~mL})$. The reference electrode was $\mathrm{Ag} / \mathrm{AgCl}, 3 \mathrm{M}$ $\mathrm{NaCl}$ (BASi, West Lafayette, IN); it was immersed in the cathode compartment. To remove residual oxygen, each compartment was fully purged with filtered argon gas using a vent filter (Whatman HEPA-vent ${ }^{\mathrm{TM}}, 0.3 \mu \mathrm{m}$ pore size). A tedlar bag was connected with a stainless steel fitting through the cathode capper to collect generated biogas. The temperature of the reactor was maintained at $37 \pm 1{ }^{\circ} \mathrm{C}$ using heating tapes (Daihan, South Korea). A constant voltage at $-0.16 \mathrm{~V}$ vs. $\mathrm{Ag} / \mathrm{AgCl}(+0.045 \mathrm{~V}$ vs. SHE) to the cathode (working electrode) was set using a multichannel potentiostat (WMPG100, WonATech, South Korea). C. pasteurianum was inoculated into the cathode compartment when the background current was stable. For glycerol fermentation, MP2 medium with glycerol ( $\sim 300 \mathrm{mM})$ instead of glucose was filled in the cathode compartment. The current was measured using the potentiostat and was continuously monitored at $4 \mathrm{~Hz}$ by an interfaced personal computer. For mature biofilm preparation, the medium was drained out under argon gas-purging after glucose depletion and then a fresh medium (glucose $\sim 100 \mathrm{mM}$ or glycerol

$\sim 300 \mathrm{mM}$ ) was refilled in the cathode compartment. The $\mathrm{pH}$ was adjusted to 6.5 and glucose or glycerol consumption by C. pasteurianum was restarted after the medium was changed.

Cyclic voltammogram. Cyclic voltammograms (CV) of suspended C. pasteurianum culture were obtained using a WMPG 1000 potentiostat/galvanostat (WonATech Co. Ltd., South Korea) with a glassy carbon working electrode (3 mm diameter, BASi, West Lafayette, IN), a platinum counter electrode, and an $\mathrm{Ag} / \mathrm{AgCl}(3 \mathrm{M} \mathrm{NaCl}, \mathrm{BASi}$ ) reference electrode. Supernatant of the cell cultures was obtained by centrifugation at $3,350 \times \mathrm{g}$ for $15 \mathrm{~min}$ and the $\mathrm{CV}$ of the supernatant was checked using glassy carbon. The CV of the biofilm on the graphite felt electrode was directly analyzed in the $\mathrm{H}$ type reactor.

Confocal laser scanning microscopy (CLSM). The structure of the biofilm on the graphite felt electrode was visualized with confocal laser scanning microscopy (CLSM). After the end of the glucose fermentation, the cathode was removed and cut it into pieces with dimensions of $\sim 3 \mathrm{~mm} \times 3 \mathrm{~mm}$. To allow for comparison, no electricity was applied to the same BES system and a piece of graphite felt was cut out Suspended cells and residual medium in the piece of graphite felt were removed by absorbing them with Kimwipes ${ }^{\circledR}$ (Kimberly-Clark, Roswell, GA) several times. Cells were stained using the LIVE/DEAD ${ }^{\circledR}$ BacLight $^{\mathrm{TM}}$ Bacterial Viability Kit (L-7012, Life Technologies, Grand Island, NY) and incubated for $15 \mathrm{~min}$ at room temperature in the dark. Each piece was rinsed out using MES buffer ( $\mathrm{pH} 5$ ) to remove excess dye and wiped with paper. Slides were analyzed and then imaged using a CLSM (Fluoview FV1000, Olympus, Tokyo, Japan) at $100 \times$ magnification, using excitation/emission wavelength of $488 / 520 \mathrm{~nm}$ and $543 / 619 \mathrm{~nm}$ for SYTO-9 and PI, respectively. The three-dimensional architecture of the biofilm was assessed by considering the $\mathrm{z}$-stack images. Images were analyzed with ImageJ software (version 1.47), a free, Java-based image processing package available at http://rsb.info.nih.gov/ij/, or using Fluoview software FV10-ASW version 2.0.

Scanning electron microscopy (SEM) and transmission electron microscopy (TEM). The pretreatment for specimen fixation was slightly modified from those previously reported ${ }^{36}$. Small pieces of cathode electrodes with biofilms were taken from the cathodes in BES and control (open circuit), and immersed in $0.1 \mathrm{M}$ phosphate buffer ( $\mathrm{pH} 7.2$ ) containing $2 \%$ (v/v) glutaradehyde for $2 \mathrm{hrs}$. After washing these pieces twice with $0.1 \mathrm{M}$ phosphate buffer, samples were dehydrated by sequential exposure in $50 \%, 70 \%, 80 \%, 90 \%, 95 \%, 100 \%$, and $100 \%$ ethanol for $15 \mathrm{~min}$ each. Air-dried sample was coated with platinum and examined with an SEM (Nova NanoSEM 200 scanning electron microscope, FEI Company) at $10 \mathrm{kV}$.

The transection image of the cells was characterized using cryo-transmission electron microscopy (FEI Tecnai G2 F20, Hilllsboro, OR) operated at $200 \mathrm{kV}$. Cells were directly collected in $2 \%(\mathrm{v} / \mathrm{v})$ glutaradehyde by squeezing graphite felts using sanitary hands when the reaction was finished.

Zeta potential. The charge of the bacterial cells was analyzed using a zeta potential analyzer. After $16 \mathrm{hr}$ incubation, squeezed cells from the cathode were collected in a centrifuged tube and quickly and gently washed with a $\mathrm{pH} 5$ phosphate buffer three times to remove residual graphite felt pieces and to rinse the cells. The O.D. of the cell used for the zeta potential analysis was $\sim 1.6$. The zeta potential was immediately determined using a Zetasizer Nano ZS (Malvern instrument, UK); average was calculated from 15 times-repeated measurements.

Quantitative determination of intracellular NADH/NAD ${ }^{+}$. To see whether intracellular $\mathrm{NAD}^{+}$reduction was induced by a cathode, NADH content was measured with $\mathrm{NAD}^{+}$level as NADH/NAD ${ }^{+}$using an Enzychrome ${ }^{\mathrm{TM}} \mathrm{NAD}^{+} / \mathrm{NADH}$ Assay kit (ECND-100, BioAssay systems, Hayward, CA) according to the 
manufacturer's instructions with $1 \mathrm{~mL}$ of each sample. The assay kit is $\mathrm{NAD}^{+}$specific and it is not interfered with by $\mathrm{NADP}^{+}$.

Analysis and calculation. Butyrate, butanol, ethanol, and acetate were analyzed using a gas chromatography (GC, Agilent technology 6890 N Network GC System, Santa Clara, CA) equipped with an HP-INNOWax column $(30 \mathrm{~m} \times 250 \mu \mathrm{m} \times 0.25 \mu \mathrm{m}$, Agilent Technologies) and a flame-ionized detector. Glucose, glycerol, and lactate were quantified using a high performance liquid chromatography HPLC (Hi-plex H, Agilent) with $5 \mathrm{mM}$ sulfuric acid as the mobile phase and a flow rate of $0.5 \mathrm{~mL} \mathrm{~min}{ }^{-1}$. Microbial growth was checked by absorbance at $600 \mathrm{~nm}$ using a Shimadzu UVmini1240 spectrophotometer (Kyoto, Japan). For a purpose of electron and carbon balance calculation, final biomass was checked by chemical oxygen demand (COD, $\mathrm{mg} / \mathrm{L}$ unit) induced by subtract soluble COD from total COD using COD vials (Cmac, South Korea, concentration range of $10-1,500 \mathrm{mg} / \mathrm{L})^{37}$. The composition of hydrogen and carbon dioxide in the biogas was analyzed using GC-TCD (Agilent Technologies $6890 \mathrm{~N}$ ).

Electron distribution value of carbon sources and the final products in BES and in the control were calculated by converting each compound to $\mathrm{e}^{-}$equivalent unit by multiplying $\mathrm{e}^{-}$equivalent number per mol by mol of each compound. Each $\mathrm{e}^{-}$ equivalent number per mol is $24 \mathrm{e}^{-}, 14 \mathrm{e}^{-}, 24 \mathrm{e}^{-}, 20 \mathrm{e}^{-}, 16 \mathrm{e}^{-}$, and $8 \mathrm{e}^{-}$for glucose, glycerol, butanol, butyrate, 1,3-PD, and acetate, respectively (see Supplementary Table 3).

Electrons consumed by microbes $(q)$ were calculated using Equation (1) with I, the current (A) and F, the Faraday constant $\left(96,485 \mathrm{C} / \mathrm{mol} \mathrm{e}^{-}\right)^{12}$.

$$
\mathrm{q}\left(\mathrm{mmol} \mathrm{e} \mathrm{e}^{-}\right)=\frac{\int \mathrm{Idt}}{\mathrm{F}}
$$

$\mathrm{NADH}$ consumption for each metabolite was calculated as shown in Equation (2) (Supplementary Tables 5, 6).

$$
\text { NADH consumption }(\mathrm{mmol})=\mathrm{n} \Delta \mathrm{P}
$$

In Equation (2), ' $\mathrm{n}$ ' is the respective number of $\mathrm{NADH}$ requirement per mole of each metabolite (4, 2, 1, and 0 for butanol, butyrate, 1,3-PD, and acetate, respectively); $\Delta \mathrm{P}$ $(\mathrm{mmol})$ is the production of each final metabolite.

NADH generation was calculated as the theoretically generated NADH on the basis of substrate consumption toward pyruvate production ( $2 \mathrm{NADH} /$ glucose; 2 $\mathrm{NADH} /$ glycerol) and electron consumption (1 NADH/2 e-) as shown in Equation (3) (Supplementary Tables 5, 6).

$$
\begin{aligned}
& \text { NADH generation }(\mathrm{mmol})=2 \Delta \mathrm{S} \text { (from substrate) or } \\
& q / 2 \text { (from electricity) }
\end{aligned}
$$

In Equation (3), $\Delta S$ (where $S$ is glucose or glycerol) is the consumption of substrate toward pyruvate production. In glycerol fermentation, $\Delta \mathrm{S}$ was determined by subtracting 1,3-PD production from glycerol consumption because NADH generation does not occur in the glycerol to $1,3-\mathrm{PD}$ pathway.

1. Du, Z., Li, H. \& Gu, T. A state of the art review on microbial fuel cells: A promising technology for wastewater treatment and bioenergy. Biotechnol Adv 25, 464-482 (2007).

2. Rosenbaum, M. \& Franks, A. Microbial catalysis in bioelectrochemical technologies: status quo, challenges and perspectives. Appl Microbiol Biotechnol 98, 509-518 (2014).

3. Rabaey, K. \& Rozendal, R. A. Microbial electrosynthesis - revisiting the electrical route for microbial production. Nat Rev Microbiol 8, 706-716 (2010).

4. Nevin, K. P., Woodard, T. L., Franks, A. E., Summers, Z. M. \& Lovley, D. R. Microbial electrosynthesis: Feeding microbes electricity to convert carbon dioxide and water to multicarbon extracellular organic compounds. mBio 1, e00103-00110 (2010).

5. Nevin, K. P. et al. Electrosynthesis of organic compounds from carbon dioxide is catalyzed by a diversity of acetogenic microorganisms. Appl Environ Microbiol 77, 2882-2886 (2011).

6. Lovley, D. R. Electromicrobiology. Annu Rev Microbiol 66, 391-409 (2012).

7. Emde, R. \& Schink, B. Enhanced propionate formation by Propionibacterium freudenreichii subsp. Freudenreichii in a three-electrode amperometric culture system. Appl Environ Microbiol 56, 2771-2776 (1990).

8. Hongo, M. \& Iwahara, M. Application of electro-energizing method to L-glutamic acid fermentation. Agric Biol Chem 43, 2075-2081 (1979).

9. Choi, O., Um, Y. \& Sang, B.-I. Butyrate production enhancement by Clostridium tyrobutyricum using electron mediators and a cathodic electron donor Biotechnol Bioeng 109, 2494-2502 (2012).

10. Kim, T. \& Kim, B. Electron flow shift in Clostridium acetobutylicum fermentation by electrochemically introduced reducing equivalent. Biotechnol Lett 10, 123-128 (1988).

11. Van Eerten-Jansen, M. C. A. A. et al. Bioelectrochemical production of caproate and caprylate from acetate by mixed cultures. ACS Sustain Chem Eng 1, 513-518 (2013)

12. Zhou, M., Chen, J., Freguia, S., Rabaey, K. \& Keller, J. Carbon and electron fluxes during the electricity driven 1,3-propanediol biosynthesis from glycerol. Environ Sci Technol 47, 11199-11205 (2013).
13. Heyndrickx, M., Vos, P. D. \& Ley, J. D. Fermentation characteristics of Clostridium pasteurianum LMG 3285 grown on glucose and mannitol. J Appl Microbiol 70, 52-58 (1991).

14. Dabrock, B., Bahl, H. \& Gottschalk, G. Parameters affecting solvent production by Clostridium pasteurianum. Appl Environ Microbiol 58, 1233-1239 (1992).

15. Carnahan, J. E., Mortenson, L. E., Mower, H. F. \& Castle, J. E. Nitrogen fixation in cell-free extracts of Clostridium pasteurianum. Biochim Biophys Acta 44, 520-535 (1960).

16. Gao, W. \& Francis, A. J. Reduction of uranium (VI) to uranium (IV) by Clostridia. Appl Environ Microbiol 74, 4580-4584 (2008).

17. Kim, T. S. \& Kim, B. H. Electron flow shift in Clostridium acetobutylicum fermentation by electrochemically introduced reducing equivalent. Biotechnol Lett 10, 123-128 (1988)

18. Kim, G. T., Webster, G., Wimpenny, J. W. T., Kim, B. H., Kim, H. J. \& Weightman, A. J. Bacterial community structure, compartmentalization and activity in a microbial fuel cell. J Appl Microbiol 101, 698-710 (2006).

19. Kim, B. H., Kim, H. J., Hyun, M. S. \& Park, D. H. Direct electrode reaction of Fe(III)-reducing bacterium, Shewanella putrefaciens. J Microbiol Biotechnol 9, 127-131 (1999).

20. Candelaria, S. L., Chen, R., Jeong, Y.-H. \& Cao, G. Highly porous chemically modified carbon cryogels and their coherent nanocomposites for energy applications. Energy Environ Sci 5, 5619-5637 (2012).

21. Daniel, E. R., Jeffrey, M. F., Daniel, B. B., Jeffrey, A. G. \& Daniel, R. B. Towards electrosynthesis in shewanella: Energetics of reversing the mtr pathway for reductive metabolism. PLoS ONE 6 (2011).

22. Marshall, C. W. \& May, H. D. Electrochemical evidence of direct electrode reduction by a thermophilic Gram-positive bacterium, Thermincola ferriacetica. Energy Environ Sci 2, 699-705 (2009).

23. Rollefson, J. B., Stephen, C. S., Tien, M. \& Bond, D. R. Identification of an extracellular polysaccharide network essential for cytochrome anchoring and biofilm formation in Geobacter sulfurreducens. J Bacteriol 193, 1023-1033 (2011).

24. Korenevsky, A. \& Beveridge, T. J. The surface physicochemistry and adhesiveness of Shewanella are affected by their surface polysaccharides. Microbiology 153 1872-1883 (2007).

25. She, P., Song, B., Xing, X.-H., Loosdrecht, M. v. \& Liu, Z. Electrolytic stimulation of bacteria Enterobacter dissolvens by a direct current. Biochem Eng J 28, 23-29 (2006).

26. Moon, C., Hwan Lee, C., Sang, B.-I. \& Um, Y. Optimization of medium compositions favoring butanol and 1,3-propanediol production from glycerol by Clostridium pasteurianum. Bioresour Technol 102, 10561-10568 (2011).

27. Milliken, C. \& May, H. Sustained generation of electricity by the spore-forming, Gram-positive, Desulfitobacterium hafniense strain DCB2. Appl Microbiol Biotechnol 73, 1180-1189 (2007).

28. Hawkins, A. S. et al. Extremely thermophilic routes to microbial electrofuels. ACS catal 1, 1043-1050 (2011).

29. Conrado, R., Haynes, C., Haendler, B. \& Toone, E. Electrofuels: A new paradigm for renewable fuels. Springer New York (2013).

30. Hawkins, A. S., McTernan, P. M., Lian, H., Kelly, R. M. \& Adams, M. W. W Biological conversion of carbon dioxide and hydrogen into liquid fuels and industrial chemicals. Curr Opin Biotechnol 24, 376-384 (2013).

31. Childers, S. E., Ciufo, S. \& Lovley, D. R. Geobacter metallireducens accesses insoluble Fe(iii) oxide by chemotaxis. Nature 416, 767-769 (2002).

32. Rabaey, K. \& Verstraete, W. Microbial fuel cells: novel biotechnology for energy generation. Trends Biotechnol 23, 291-298 (2005).

33. Rosenbaum, M. A. \& Henrich, A. W. Engineering microbial electrocatalysis for chemical and fuel production. Curr Opin Biotechnol 29, 93-98 (2014).

34. Biebl, H. Fermentation of glycerol by Clostridium pasteurianum - batch and continuous culture studies. J Ind Microbiol Biotechnol 27, 18-26 (2001).

35. Baer, S. H., Blaschek, H. P. \& Smith, T. L. Effect of butanol challenge and temperature on lipid composition and membrane fluidity of butanol-tolerant Clostridium acetobutylicum. Appl Environ Microbiol 53, 2854-2861 (1987).

36. Chung, K. \& Okabe, S. Continuous power generation and microbial community structure of the anode biofilms in a three-stage microbial fuel cell system. Appl Microbiol Biotechnol 83, 965-977 (2009).

37. Lee, H.-S., Krajmalinik-Brown, R., Zhang, H. \& Rittmann, B. E. An electron-flow model can predict complex redox reactions in mixed-culture fermentative $\mathrm{BioH}_{2}$ : Microbial ecology evidence. Biotechnol Bioeng 104, 687-697 (2009).

\section{Acknowledgments}

This research was supported by the KIST Institutional Program (Project No. 2E24500) and the Creative Allied Project (CAP) of the Korea Research Council of Fundamental Science and Technology (KRCF)/Korea Institute of Science and Technology (KIST) (Project No. 2E24832)

\section{Author contributions}

O.C., H.M.H. and Y.U. conceived and coordinated the experiments. O.C. performed the experiments. O.C. and Y.U. wrote the manuscript. T.K. conducted and analyzed the confocal microscopy images under the supervision of O.C. 


\section{Additional information}

Supplementary information accompanies this paper at http://www.nature.com/ scientificreports

Competing financial interests: The authors declare no competing financial interests. How to cite this article: Choi, O., Kim, T., Woo, H.M. \& Um, Y. Electricity-driven metabolic shift through direct electron uptake by electroactive heterotroph Clostridium pasteurianum. Sci. Rep. 4, 6961; DOI:10.1038/srep06961 (2014).
(1) (2) This work is licensed under a Creative Commons Attribution-NonCommercialShareAlike 4.0 International License. The images or other third party material in this article are included in the article's Creative Commons license, unless indicated otherwise in the credit line; if the material is not included under the Creative Commons license, users will need to obtain permission from the license holder in order to reproduce the material. To view a copy of this license, visit http:// creativecommons.org/licenses/by-nc-sa/4.0/ 